\title{
Identification of AtSM34, a novel tonoplast intrinsic protein- interacting polypeptide expressed in response to osmotic stress in germinating seedlings
}

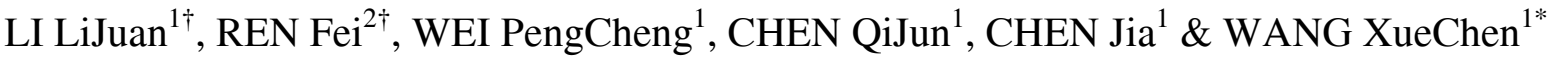 \\ ${ }^{1}$ State Key Laboratory of Plant Physiology and Biochemistry, College of Biological Sciences, China Agricultural University, Beijing 100193, \\ China; \\ ${ }^{2}$ Institute of Forestry and Pomology, Beijing Academy of Agriculture and Forestry Sciences, Beijing 100093, China
}

\begin{abstract}
Aquaporins are implicated in a wide variety of plant physiological processes, although the mechanisms involved in their regulation are not fully understood. To gain further insight into the regulatory factors involved in this process, we used a yeast two-hybrid system to screen for potential binding partners to the Arabidopsis tonoplast intrinsic protein (TIP) AtTIP1;1. This was the first protein identified to be associated with high water permeability in vacuolar membranes from Arabidopsis thaliana. Using AtTIP1;1 as bait, a novel binding protein was identified in both yeast and plant cells. This prey protein, named AtSM34, was a 309 aa polypeptide with a predicted molecular mass of $34 \mathrm{kD}$ and contained a single MYB/SANT-like domain. AtSM34 promoter:: GUS histochemical staining analysis detected AtSM34 expression in flowers, stems and leaves, particularly in the vascular tissues, in response to osmotic stress. AtSM34 expression was localized in the endoplasmic reticulum membrane, and sequence deletion analysis revealed that the N-terminal coding region (amino acids 1-83) was critical for this localization. Overexpression of AtSM34 resulted in hypersensitivity to exogenous mannitol, sorbitol and abscisic acid, and caused a significant delay in germination. AtSM34 interacted with AtTIP1;2 and AtTIP2;1, which are essential proteins for modulation of tonoplast permeability and highly expressed in germinating seedlings. These data indicate AtSM34 is a novel TIPs binding protein involved in the osmotic stress response of seedlings at an early stage of development.
\end{abstract}

Arabidopsis, osmotic stress, tonoplast intrinsic proteins, endoplasmic reticulum

Citation: Li L J, Ren F, Wei P C, et al. Identification of AtSM34, a novel tonoplast intrinsic protein-interacting polypeptide expressed in response to osmotic stress in germinating seedlings. Chinese Sci Bull, 2011, 56: 3518-3530, doi: 10.1007/s11434-011-4793-4

Aquaporins are channel proteins involved in the regulation of water permeability of cellular membranes [1] and are actively involved in plant growth and development. Regulation of these proteins and their integrated functions is important for the maintenance of water balance under conditions of stress [2].

Aquaporin activity is regulated by factors that include phosphorylation, heteromerization, $\mathrm{pH}, \mathrm{Ca}^{2+}$, temperature and solute gradients, which modify their gating behavior [3-6]. Recent reports have suggested other membrane-

$\dagger$ These authors contributed equally to this work

*Corresponding author (email: xcwang@ cau.edu.cn) associated or cytosolic proteins might be involved in the regulation of aquaporin expression, activity and trafficking or might function as multicomponent protein complexes through protein-protein interactions. There are a number of examples of such interactions in animals and plants. These include the $\mathrm{Ca}^{2+}$-dependent binding of two calmodulin molecules with a single aquaporin-0 tetramer, which results in the temporal regulation of channel activity [7], and the interaction of heat shock protein 70 (Hsp70) in the regulation of aquaporin-2-mediated trafficking in rat kidney cells [8]. In plants, the Cucumber mosaic virus (CMV) 1a interacts both with TIP1 and TIP2, which possibly affects CMV replication via interactions in the tonoplasts [9]. Further- 
more, soybean cytosolic glutamine synthetase interacts with the C-terminal domain of the symbiosome membrane nodulin 26 (NOD26), which is thought to be localized at the cytosolic side of the symbiosome membrane and to promote efficient assimilation of fixed nitrogen and prevent ammonia toxicity [10]. These protein interactions also regulate the amount and localization of aquaporins in response to environmental stress factors. Overexpression of hot pepper RmalH1, a homolog of E3 ubiquitin ligase, resulted in reduction of the level of AtPIP2;1 and inhibited trafficking of AtPIP2;1 from the endoplasmic reticulum (ER) to the plasma membrane in protoplasts. Downregulation of plasma membrane aquaporin levels conferred tolerance to dehydration in transgenic Arabidopsis plants [11].

In plants, vacuoles are unique and essential organelles with important roles in turgor regulation, osmotic adjustment, cell signaling, storage and digestion. Tonoplast intrinsic proteins (TIPs) were identified as a tonoplast-localized water channel subgroup [12]. Variations in TIP expression levels might influence water movement across the tonoplast and might also affect the responses of plants to abiotic stress [13]. The effects of osmotic stress were investigated in ice plants (Mesembryanthemum crystallinum). Mannitol-induced water imbalance increased expression of McTIP1;2 (McMIPF) proteins and resulted in its redistribution to the endosomal compartment [14], which implicates these changes in response to osmotic stress.

AtTIP1;1 belongs to the Arabidopsis thaliana TIP subfamily and was one of the first proteins identified with high water permeability when expressed in Xenopus oocytes [15]. However, AtTIP1;1 knock-out lines or a double knockout for both TIP1;1 and TIP1;2 in Arabidopsis showed no apparent alterations in macroscopic phenotype. This is likely to be because of the functional redundancy of other TIP homologs [16,17]. These findings indicated undetected functions or regulatory mechanisms are associated with AtTIP1;1.

This study focused on the identification of AtTIP1;1 interacting proteins with the aim of gaining a better understanding of the function of AtTIP1;1. Using AtTIP1;1 as bait in a split ubiquitin yeast screening system, a novel interacting protein was identified and the interaction was confirmed through bimolecular fluorescence complementation (BiFC) studies. This novel protein encoded a putative 309 amino acid (aa) polypeptide with a predicted molecular mass of $34 \mathrm{kD}$ that contained a single MYB/SANT-like domain. The protein was named AtSM34 to reflect this, and it was also inferred as maMYB [18], although it was not among the typical $M Y B$ genes that have been identified in $A$. thaliana [19]. Overexpressing lines were hypersensitive to osmotic stress during germination and early seedling growth. Further study showed expression to be localized in the ER, for which the N-terminal was critical. Subsequent experiments revealed that AtSM34 also interacted with the other TIP isoforms, AtTIP1;2 and AtTIP2;1 which are most abundant in germinating seedlings. These observations indicated interactions between AtSM34 and AtTIPs might affect the natural expression patterns of members of TIP subfamilies during Arabidopsis seed germination.

\section{Materials and methods}

\subsection{Plant material and growth conditions}

Arabidopsis thaliana ecotype Col-0 was used as the wild type for all experiments. The transgenic lines that expressed $35 S:: s p R F P-A F V Y$ (the C-terminal vacuolar sorting signal of phaseolin, the tetrapeptide AFVY, fused to the C-terminus of the red fluorescent protein [RFP] sequence) were kindly provided by Dr. Lorenzo Frigerio (University of Warwick). Arabidopsis thaliana seeds were surface-sterilized in $0.5 \%$ sodium hypochlorite solution for $15 \mathrm{~min}$, then washed 5 times with sterile water. Sterilized seeds were sown on half-strength Murashige and Skoog (1/2 MS) medium (Sigma, St Louis, MO, USA), which contained 1\% (w/v) sucrose and $0.5 \%$ phytagel (Sigma), adjusted to $\mathrm{pH}$ 5.8. The plates were incubated in the dark for $3 \mathrm{~d}$ at $4^{\circ} \mathrm{C}$ before transferal to growth chambers under long-day conditions with fluorescent illumination (16 h light/8 h dark) at $22-23^{\circ} \mathrm{C}$.

For RNA analysis, 6-d-old seedlings were grown on $1 / 2$ MS medium supplemented with $1 \%$ (w/v) sucrose. Seedlings were treated with $1 / 2$ MS liquid medium supplemented to provide the stress conditions investigated.

\subsection{Yeast split-ubiquitin system screening and interac- tion assays}

DUAL membrane technology was used to screen for proteins that interacted with membrane-associated AtTIP1;1. The TIP $1 ; 1$ coding region was cloned into the $S f i$ I site of the pTMBV4 bait vector. The following primers were used: TIP1Forward (5'-GGCCATTACGGCCTTAAAAATGCCGATCAGAAACATC-3') and TIP1Reverse (5'-GGCCGAGGCGGCCCCGTAGTCTGTGGTTGGGAGCT-3').

An A. thaliana NubG-x cDNA library (P02210) was screened to identify protein interaction partners of AtTIP1;1. The yeast strain DSY-1 was used for cDNA library screening, and yeast transformation was performed according to the manufacturer's protocol (DUALsystems Biotech, Schlieren, Switzerland). Transformants were plated onto synthetic dropout (SD) medium lacking leucine (Leu), tryptophan (Trp), and histidine (His) supplemented with $10 \mathrm{mmol} / \mathrm{L}$ 3-amino-1,2,4-triazole (3-AT). Positive clones were replated on the SD selection medium and analyzed for $\beta$-galactosidase activity. Plasmids of the positive blue colonies were recovered from yeast, transformed into $E$. coli, and sequenced. To confirm the positive interactions, isolated prey plasmids were transformed into the yeast strain DSY1 with the bait pTMBV4-TIP $1 ; 1$ or control bait $p M B V$-Alg5. 


\subsection{Bimolecular fluorescence complementation assay and confocal imaging}

The BiFC assay was performed as previously described [20]. The primers used in this experiment were: TIP1-BForward: 5'-GCTCTAGAATGCCGATCAGAAACATCG-3'; TIP1BReverse: 5'-CGGGATCCGTAGTCTGTGGTTGGGAGCT-3'; TIP12-BForward: 5'-GCTCTAGAATGCCGACCAGAAACATCG-3'; TIP12-BReverse: 5'-CGGGATCCGTAATCGGTGGTAGGCAATTG-3'; TIP21-BForward: 5'GCTCTAGAATGGCTGGAGTTGCCTTTGG-3'; TIP21BReverse: 5'-GGGGTACCGAAATCAGCAGAAGCAAGAGG-3'; SM34-BForward: 5'-GCTCTAGAATGGATTTTTTCGACGAAGAC-3'; SM34-BReverse: 5'-CGGGATCCATTAGCTGGAGTTTTCGAGC-3'; SM34-250BFor-ward: 5'-GCTCTAGAATGGGTAAGATTCGCGTCGGTT-3' (Restriction enzyme recognition sites are underlined).

PCR-amplified AtTIP1;1, AtTIP1;2 and AtTIP2;1 coding regions were cloned into pUC-SPYCE vectors [20] and sequenced. The AtSM34 full-length coding region and the fragment with a deletion of the N-terminal amino acids 1-83 (AtSM34483aa) were inserted into pUC-SPYNE [20] and sequenced. Arabidopsis mesophyll protoplasts were transformed with the resultant constructs, $p U C$-SPYNEAtSM34 $483 a a$ and $p U C$-SPYNE-AtSM34, or the corresponding control by PEG-induced DNA uptake [21]. All transformations were repeated at least three times using Arabidopsis mesophyll protoplasts isolated from different batches of plants. FM4-64 (N-(3-triethylammoniumpropyl)-4-(6-(4(diethylamino) phenyl) hexatrienyl) pyridinium dibromide; Invitrogen, Eugene, OR, USA) was dissolved in DMSO at 1 $\mathrm{mmol} / \mathrm{L}$ and diluted in W5 solution. For labeling, the transformed Arabidopsis mesophyll protoplasts were stained with $15 \mu \mathrm{mol} / \mathrm{L}$ FM4-64 for $2 \mathrm{~h}$ and washed with W5 solution three times before observations were performed.

Image analysis was performed by confocal laser scanning microscopy (LSM 510 META, Zeiss, Jena, Germany) under a Zeiss $\times 40$ oil objective (Plan-NEOFLUAR, NA 1.3). The excitation wavelength for YFP detection was $488 \mathrm{~nm}$ and emissions were collected between 515 and $530 \mathrm{~nm}$. FM4-64 and RFP detection were excited at $543 \mathrm{~nm}$ and emissions were collected between 585 and $615 \mathrm{~nm}$. Fluorescence was recorded in the multi-track mode. All confocal images were processed using LSM Image Browser version 3.2 (Zeiss) and exported as TIFF files.

\subsection{Isolation of the AtSM34 promoter region and his- tochemical GUS assays}

To examine the tissue-specific expression of AtSM34, a region containing 1035 base pairs (bp) upstream of the initiation codon (ATG) of the AtSM34 promoter region was amplified by PCR from Arabidopsis genomic DNA using KOD DNA polymerase. The following primers were used: Forward (5'-CGCGGATCCTACTCAAAGCAAACAAAC-
GAAG-3') and Reverse (5'-GGCGAATTCTGTTGCTCTGTTGCACTGTAGA-3') (restriction enzyme recognition sites are underlined).

Integrity of the amplified product was confirmed by sequencing, then the fragment was cloned into the BamHI and EcoRI sites of the pCAMBIA1391 vector [22]. The construct was introduced into Agrobacterium tumefaciens strain GV3101 and used to transform Arabidopsis Col-0. GUS staining was performed as described previously [22]. Finally, seedlings were washed with $70 \%$ ethanol to remove chlorophyll.

\subsection{Subcellular localization of the AtSM34-GFP fusion protein}

The full-length open reading frame encoding region of

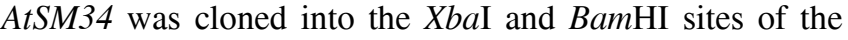
transient expression vector pUC-EGFP [23] downstream from the Cauliflower mosaic virus (CaMV) $35 \mathrm{~S}$ promoter. The AtSM34 $483 a a$ fragment was excised from $p U C$ SPYNE-AtSM34 $833 a a$ using the $X b a \mathrm{I} / B a m H I$ enzymes, and then cloned into pUC-EGFP.

The AtSM34 coding region was amplified by PCR and fused to the C-terminus of GFP driven by the CMV 35S promoter in the modified pCAMBIA1300 vector [24] using the SpeI and SalI sites. The primers used were as follows: SM34NGF (5'-GACTAGTATGGATTTTTTCGACGAAG$\left.3^{\prime}\right)$ and SM34NGR (5'-ACGCGTCGACTTAATTAGCTGGAGTTTT-3') (restriction enzyme recognition sites are underlined). Arabidopsis mesophyll protoplasts were transformed by PEG-induced DNA uptake [21].

For stable expression, the AtSM34 coding region fragment fused to GFP was cloned into the vector pBI121 [25] and expressed under the control of the $35 \mathrm{~S}$ promoter. This was introduced into Arabidopsis plants through A. tumefaciens strain GV3101-mediated transformation. The roots of kanamycin-resistant seedlings from transgenic plants containing the 35S::AtSM34-GFP fusion protein were analyzed by direct confocal microscopy. Five independent 35S:: AtSM34-GFP transgenic lines were generated and used to investigate the localization of the fusion protein. The excitation wavelength for GFP detection was $488 \mathrm{~nm}$ and emissions were collected between 515 and $530 \mathrm{~nm}$ under a Zeiss $\times 40$ oil objective. To visualize the ER, the transformed $A r$ abidopsis mesophyll protoplasts and roots of 35S::AtSM34GFP transgenic plants were stained with $1 \mu \mathrm{mol} / \mathrm{L}$ redorange-fluorescent BODIPY 558/568-conjugated brefeldin A (B7449, Invitrogen) for $30 \mathrm{~min}$. Fluorescence was detected at an excitation wavelength of $543 \mathrm{~nm}$ and emissions collected between 585 and $615 \mathrm{~nm}$.

\subsection{Semiquantitative reverse transcription and quan-} titative real-time PCR analysis

Total RNA was extracted from the collected samples using 
a RNA purification kit (BioTeke) according to the manufacturer's instructions. Reverse transcription was performed using M-MLV reverse transcriptase (Promega, Madison, WI, USA) with an Oligo-dT18 primer (TaKaRa). The synthesized cDNA was used for semiquantitative or quantitative PCR analysis. In semiquantitative reverse transcription PCR (RT-PCR) analysis, the following AtSM34 gene-specific primers were used: 45420-171Forward (5'-TGAACCTGCCAAATCGCTAC-3') and 45420-741Reverse (5'-ACCTTCCGCATCTCCACC-3').

ACTIN2/8 (At3g18780) mRNA expression was amplified as an internal control. The specific primers used for semiquantitative RT-PCR were: 5'-TCTTCCGCTCTTTCTTTCCA-3' and 5'-GAGAGAACAGCTTGGATGGC-3' [26]. Amplification of ACTIN2/8 and AtSM34 was carried out using 23 and 29 cycles, respectively. The PCR program used was as follows: $94^{\circ} \mathrm{C}$ for $30 \mathrm{~s}, 56^{\circ} \mathrm{C}$ for $30 \mathrm{~s}$ and $72^{\circ} \mathrm{C}$ for $50 \mathrm{~s}$. A final incubation at $72^{\circ} \mathrm{C}$ for $7 \mathrm{~min}$ was performed to complete product synthesis.

Quantitative real-time PCR analysis was performed with specific primers of AtSM34 gene: 45420Forward (5'TGATGATTACGCTCAGTTT-3') and 45420Reverse (5'ACCTTCCGCATCTCCACC-3'). Quantitative real-time PCR analysis of the ACTIN2/8 genes was performed using the following specific primers: ACTIN2/8-Forward (5'GGTAACATTGTGCTCAGTGGTGG-3') and ACTIN2/8Reverse (5'-AACGACCTTAATCTTCATGCTGC-3') [27].

The relative expression levels of genes detected were calculated against a standard curve of expression of the ACTIN2/8 calibrator using the $2^{-\Delta \Delta \mathrm{C}_{\mathrm{T}}}$ method [28]. Realtime quantitative PCR experiments were independently repeated in triplicate.

\subsection{Generating AtSM34 overexpression lines}

The full-length coding region sequence of AtSM34 was amplified using Pfu DNA polymerase from Arabidopsis seedling cDNA by PCR using the following primers: 45420 Forward (5'-GCTCTAGAATGGATTTTTTCGACGAAGAC-3') and 45420Reverse (5'-GGGGTACCATTAGCTGGAGTTTTCGAG-3') (restriction enzyme recognition sites are underlined).

Amplified regions were cloned into the $X b a \mathrm{I} / K p n \mathrm{I}$ sites of the modified pSuper1300 vector [29] and sequenced. To modify the pSuper1300 vector, the FLAG fragment was amplified by PCR and inserted into the pSuper1300 vector using the KpnI and SacI sites. This construct (pSuper1300AtSM34-FLAG-TNOS) was introduced into A. tumefaciens strain GV3101 and used to transform wild-type Arabidopsis plants using the floral-dip method [30]. Transgenic plants were screened for hygromycin resistance on $25 \mu \mathrm{g} / \mathrm{mL}$ hygromycin plates. Resistant transformants were transferred to soil and grown in a greenhouse. According to the segregation ratios of $T_{2}$ and $T_{3}$ seeds on hygromycin, $T_{3}$ homozygous lines were selected for expression analysis and pheno- type characterization.

Approximately 60 seeds from each of the wild-type and AtSM34 overexpression transgenic lines (OE-6 and OE-10) were sown on $1 / 2 \mathrm{MS}$ containing $1 \%(\mathrm{w} / \mathrm{v})$ sucrose media and supplemented with mannitol (400 $\mathrm{mmol} / \mathrm{L})$, sorbitol (400 mmol/L) or abscisic acid (ABA) $(1 \mu \mathrm{mol} / \mathrm{L})$. Plants were incubated at $4{ }^{\circ} \mathrm{C}$ for $3 \mathrm{~d}$ before being placed at $22^{\circ} \mathrm{C}$ under long-day conditions. Germination (emergence of radicles) was scored daily for $6 \mathrm{~d}$. Plant growth was monitored and photographed after $7 \mathrm{~d}$. The experiments were repeated independently three times.

\subsection{Western blot analysis}

Total proteins were extracted by incubating the plant materials in protein extraction buffer. Proteins were fractionated by SDS-PAGE on $12 \%$ (w/v) gel using a minigel system (Bio-Rad, Hercules, CA, USA). The separated proteins were transferred from the gel to a polyvinylidene fluoride nitrocellulose membrane (Bio-Rad), and then blotted with an anti-flag (Sigma) primary antibody (diluted 1:10000). The membrane was washed three times with TBS-T buffer followed by incubation for $1 \mathrm{~h}$ with the secondary antibody (peroxidase-conjugated affinipure goat anti-rabbit IgG $[\mathrm{H}+\mathrm{L}])$. The washing process was repeated before the proteins on the membrane were detected with an enhanced chemiluminescence system (Amersham Biosciences, Uppsala, Sweden).

\section{Results}

\subsection{Yeast split-ubiquitin assay for the interaction be- tween AtSM34 and AtTIP1;1}

To search for potential protein interactions, AtTIP1;1 was used as bait to screen the NubG-x cDNA library from $A$. thaliana 6-d-old seedlings according to the protocol provided for the DUAL membrane yeast split-ubiquitin system (DUALsystems Biotech). Prey plasmids from the positive clones were isolated and sequenced. Further analysis identified a unique clone encoding a 309 aa putative polypeptide (AtSM34, At5g45420) with a predicted molecular mass of $34 \mathrm{kD}$ that contained a MYB/SANT-like domain.

To verify the interaction between AtSM34 and AtTIP1;1, the prey plasmid $p D L 2-N u b-A t S M 34$ was retransformed to the yeast DSY1 expressing the bait pTMBV4-TIP1;1-Cub. Transformed yeast survived on the SD selection medium $\mathrm{SD} /$-His-Trp-Leu containing $10 \mathrm{mmol} / \mathrm{L} 3-\mathrm{AT}$, and tested positive for $\beta$-galactosidase activity using the $\mathrm{X}$-gal filter test (Figure 1(a)). No interactions were observed in the negative controls $p D L 2-N u b-A t S M 34 / p M B V$-Alg5 and pTMBV4TIP1;1-Cub/pAlg5-NubG. These results provided independent evidence of the interaction between AtSM34 and TIP1;1 in the yeast cells. 

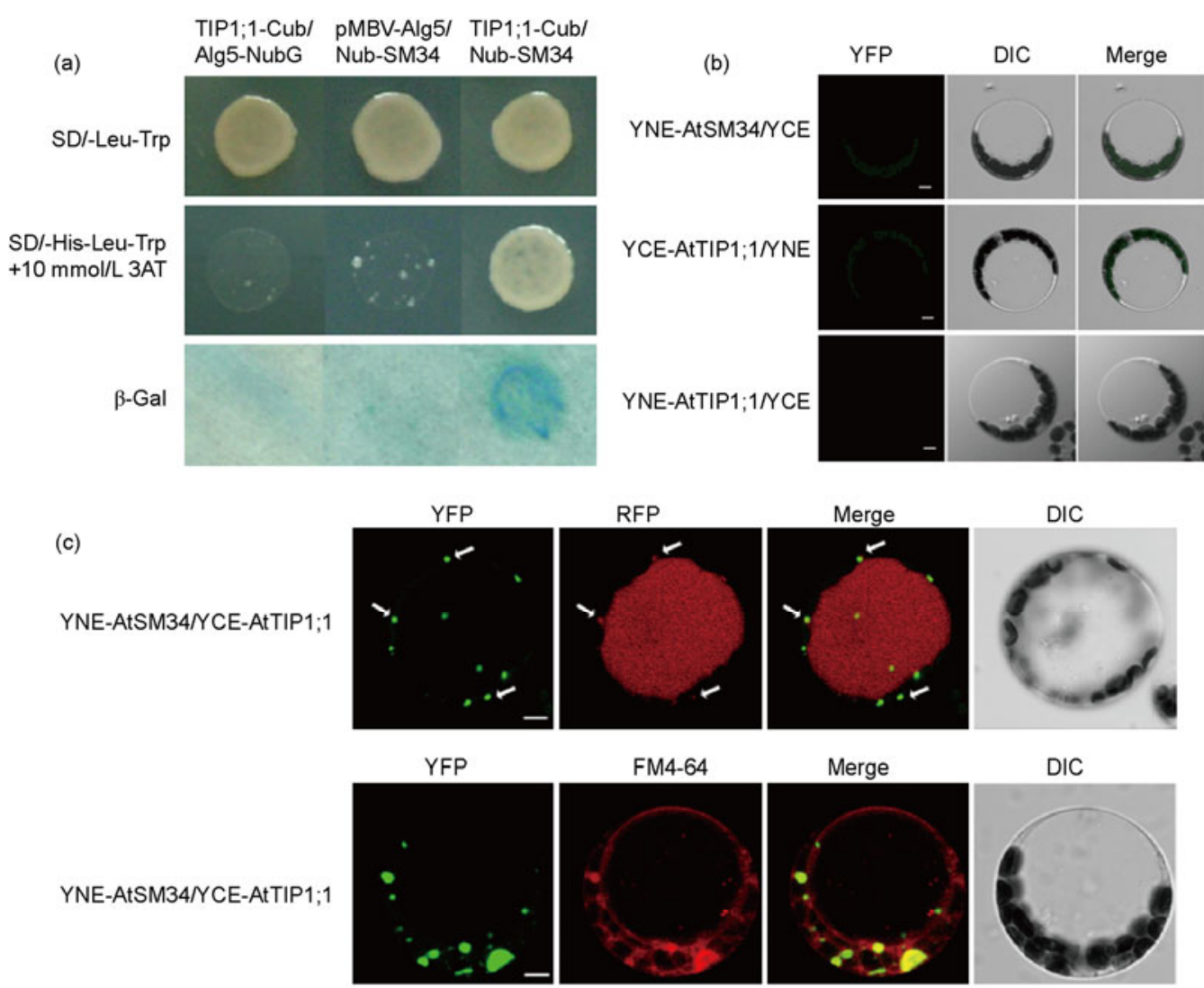

FM4-64

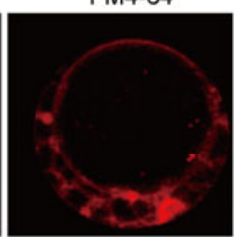

Merge

$\mathrm{DIC}$
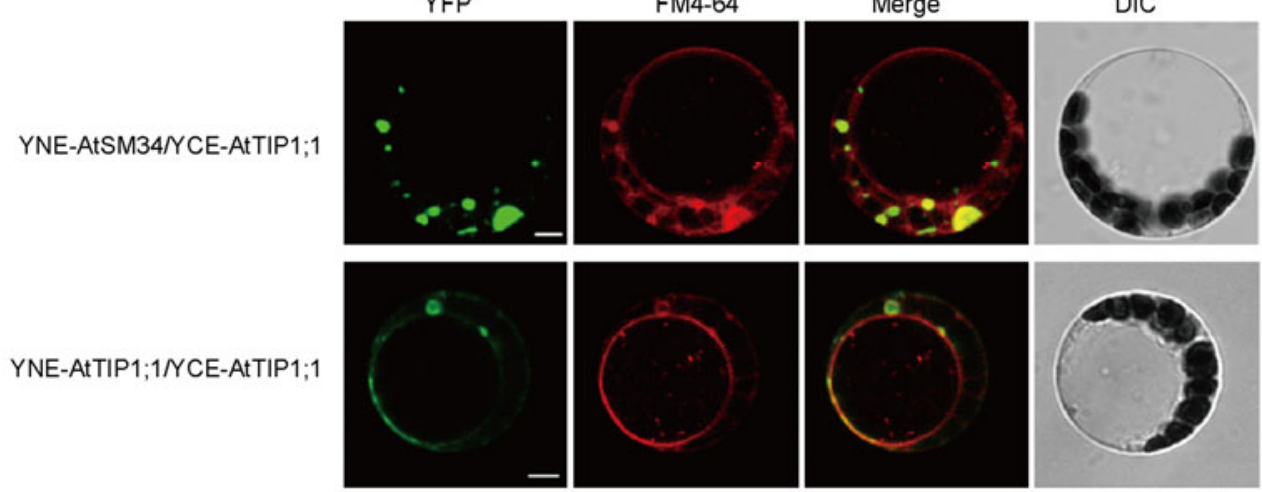

Figure 1 AtSM34 interacts with AtTIP1;1 in both yeast and Arabidopsis cells. (a) In a yeast split-ubiquitin system assay, AtSM34 interacted with AtTIP1;1 in yeast. DSY1 cells were transformed with $p T M B V 4-T I P 1 ; 1-C u b$ and $p D L 2-N u b-A t S M 34$. Transformants were cultured and then dipped at the same concentration on the plate that contained a synthetic dropout selection medium that lacked either Trp and Leu (SD/-Trp-Leu) or Trp, Leu and His (SD/-Trp-Leu-His) supplemented with $10 \mathrm{mmol} / \mathrm{L} 3$-AT. The combination of $p T M B V 4-T I P 1 ; 1-C u b / p A l g 5-N u b G$ and $p M B V-A l g 5 / p D L 2-N u b-S M 34$ were used as negative controls. Results were confirmed in at least three independent experiments. (b) YFP fluorescence detection of Arabidopsis mesophyll protoplasts cotransformed with corresponding control combinations of YNE-AtSM34/YCE, YCE-AtTIP1;1/YNE and YNE-AtTIP1;1/YCE. (c) Membrane-associated interactions between AtSM34/AtTIP1;1 and AtTIP1;1 detected by a bimolecular fluorescence complementation assay. YFP fluorescence detection of Arabidopsis mesophyll protoplasts cotransformed with YNE-AtSM34 and YCE-AtTIP1;1, or YNE-AtTIP1;1 and YCE-AtTIP1;1. The arrows indicate the YFP signals colocalized with the RFP fluorescence of small vesicles in the protoplasts of $35 S:: s p R F P-A F V Y$ transgenic lines (upper panel). BiFC assays showed the interaction between AtTIP1;1 and AtSM34 in the wild-type Arabidopsis protoplasts stained with FM-64 dye. Secretory vesicles and tonoplasts were stained red with FM-64 (middle panel). Confocal image analysis of Arabidopsis mesophyll protoplasts cotransformed with YNE-AtTIP1;1 and YCE-AtTIP1;1 stained with FM-64 dye (lower panel). YFP, yellow fluorescent protein fluorescence signals; RFP, red fluorescent protein fluorescence signals; DIC, differential interference contrast optics; Merge, the superimposition of both images. YFP fluorescence signals are shown in green. Scale bar represents $5 \mu \mathrm{m}$.

\subsection{Bimolecular fluorescence complementation assay}

The interaction of AtTIP1;1 and AtSM34 in plants was confirmed previously by BiFC assays [20]. TIP1;1 was fused with the C-terminal portion of the yellow fluorescent protein (YFP) and AtSM34 was fused with the N-terminal portion of the same protein. These plasmids were then cotransformed into the Arabidopsis 35S::spRFP-AFVY transgenic lines or wild-type Arabidopsis mesophyll protoplasts. Localized restored YFP fluorescence was detected as punctate structures similar to small vesicles or prevacuolar compartments (Figure 1(c)). Previously, 35S::spRFP-AFVY was reported to localize exclusively in the lumens of the large, central vegetative vacuoles [31]. Vacuolar fusion was dynamic, with tetrapeptide AFVY carrying the RFP proteins that could also label a variety of vesicles, such as proteinstorage vacuoles, dense vesicles or precursor-accumulating vesicles [32] concurrently. The arrows in Figure 1(c) indicate that the YFP fluorescence colocalized with the red separated small vesicles around the central large vacuole (Fig- 
ure 1(c) upper merge panel). To identify the compartments further, we also stained the wild-type Arabidopsis mesophyll protoplast with FM4-64 dye, which is a useful tool to track endocytosis and vesicle trafficking in living cells [33]. The restored YFP signals colocalized with some vesicles stained with FM 4-64 intracellularly, but were not detected on the central vacuole membrane (Figure 1(c) middle merge panel). The BiFC assays showed that the interaction between AtTIP1;1 and AtSM34 occurred in the small vesicles. These data indicate AtSM34 might be involved in vesicle trafficking.

As a comparison with the above BiFC assays, TIP1;1 was fused with both the $\mathrm{C}$ - and N-terminal portions of YFP that were expressed transiently in Arabidopsis mesophyll protoplasts simultaneously stained with FM4-64. Aquaporins are known to form homomers before they can mediate their biological functions. The restored YFP fluorescence was located preferentially in the tonoplasts in both the large central vacuole and an adjacent small peripheral vacuole (Figure 1(c) bottom panel). These findings indicated the interaction between AtTIP1;1 and AtSM34 occurred in the vesicles, but not on the membrane of the large central vacuole.

No YFP signal was detected in protoplasts transformed with the combinations of YNE-AtSM34 and YCE empty vector, YCE-AtTIP1;1 and YNE empty vector, or YNEAtTIP1;1 and YCE empty vector (Figure 1(b)).

\subsection{Expression pattern of AtSM34 in different tissues}

Expression patterns of AtSM34 in different organs of Arabidopsis plants were analyzed by RT-PCR. Total RNA was isolated from roots, stems, leaves, flowers, and young siliques. Expression of ACTIN2/8 was used as the loading control. As shown in Figure 2, the transcription levels of AtSM34 detected in the flowers, leaves and stems were higher than the levels detected in roots and young siliques.

The expression patterns of AtSM34 were examined in greater detail by generation of a construct encoding the $\beta$-glucuronidase (GUS) reporter gene under the control of the AtSM34 promoter (AtSM34promoter::GUS). Following transformation of wild-type Arabidopsis plants, more than three independent lines were used for analyses. Strong GUS signals were observed in cotyledons of the germinating seeds (Figure 2(a) indicated by white arrow) and seedlings (Figure 2(b)-(d)). High GUS activities were detected in both two-week-old seedlings (Figure 2(e)) and rosette

\begin{tabular}{|c|c|c|c|c|c|}
\hline & Root & Stem & Leaf & Flower & Silique \\
\hline AtSM34 & $\operatorname{sen} 2$ & tas & sas & 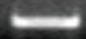 & \\
\hline ACTIN2/8 & cense & 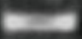 & cese & s. & 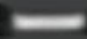 \\
\hline
\end{tabular}
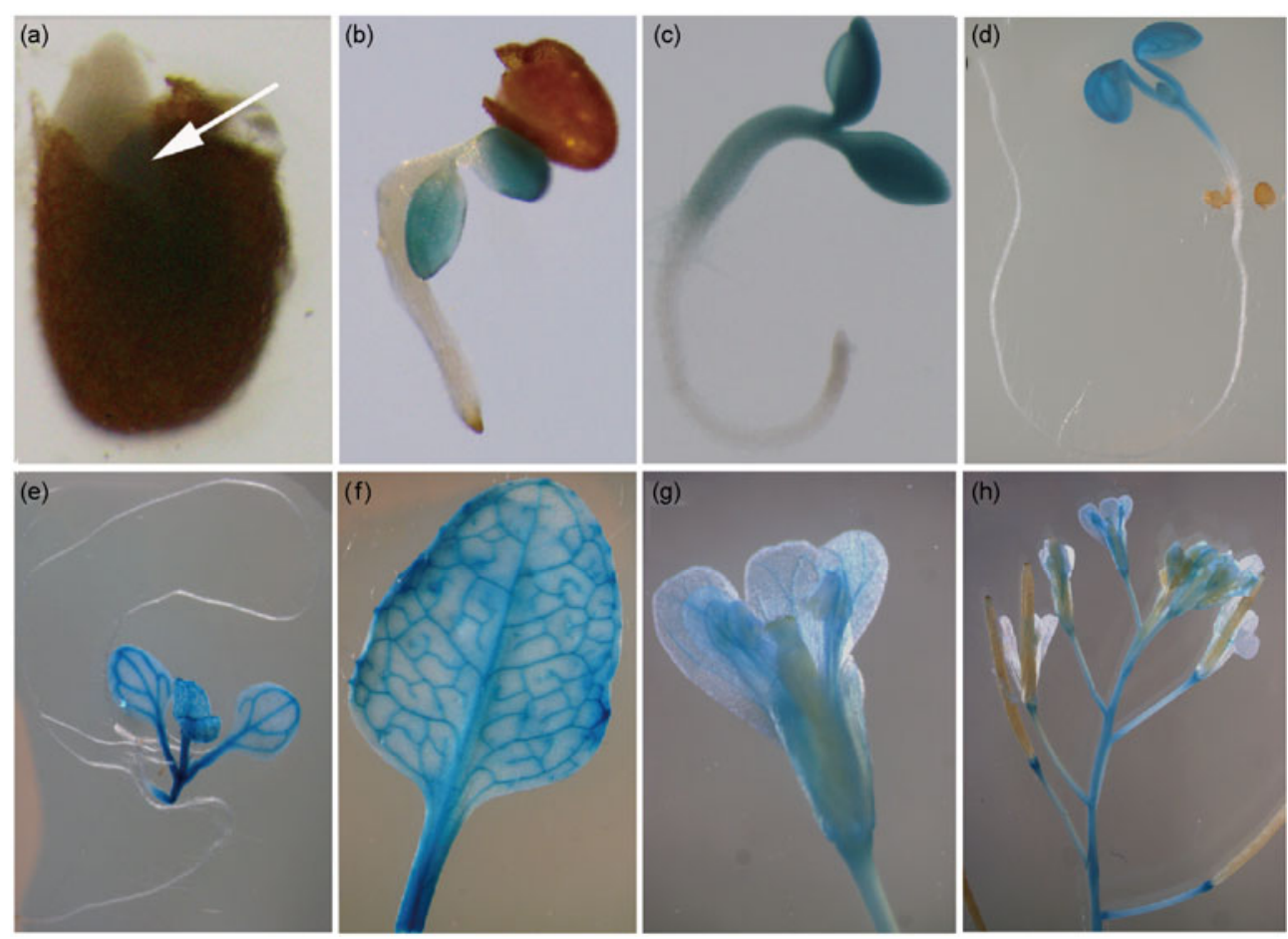

Figure 2 Expression of AtSM34. Upper panel: semiquantitative RT-PCR analysis of AtSM34 transcripts in different organs of wild-type Arabidopsis. Total RNA was isolated from different tissues (root, stem, leaf, flower, and silique) of 4-week-old Arabidopsis (ecotype Col-0) plants. RT-PCR was performed with AtSM34-specific primers. ACTIN2/8 was used as the loading control. (a)-(h) Histochemical GUS analysis of AtSM34 promoter::GUS transgenic plants. (a) Seed germinated for $24 \mathrm{~h}$; (b) 2-d-old seedling; (c) 3-d-old seedling; (d) 5-d-old seedling; (e) whole seedling (2 weeks); (f) rosette leaf (4 weeks); (g) flower (5 weeks); (h) stem and inflorescence (5 weeks). 
leaves, particularly the vascular tissues (Figure 2(f)). Strong staining was also observed in mature stems (Figure 2(h)) and in the petals and calyces of flowers (Figure 2(g)). However, GUS activity was virtually absent in the roots and young siliques.

\subsection{Localization of AtSM34 to the endoplasmic reticulum}

The Arabidopsis Information Resource (TAIR) database (http://www.arabidopsis.org) forecast exhibited AtSM34 was localized to endoplasmic reticulum. To examine the subcellular localization of AtSM34, GFP fused either at the C-terminal (AtSM34-GFP) or N-terminal (GFP-AtSM34) to
AtSM34 under the control of the CaMV 35S promoter was transiently expressed in Arabidopsis mesophyll protoplasts. Green fluorescent structures similar to the ER were observed by confocal microscopy. Previous investigations showed ER-localized proteins exhibit a network pattern in protoplasts [34]. To confirm the localization of AtSM34 expression, mesophyll protoplasts cells were stained with the ER marker red-orange-fluorescent BODIPY 558/568-conjugated brefeldin A. Overlaid green and red signals confirmed that the GFP signals were colocalized with the ER (Figure 3(a)). Appending GFP to the N- or C-terminus of AtSM34 resulted in an identical pattern of localization (Figure 3(a)).

Further studies of transgenic seedlings expressing AtSM34-

35:::AtSM34-GFP
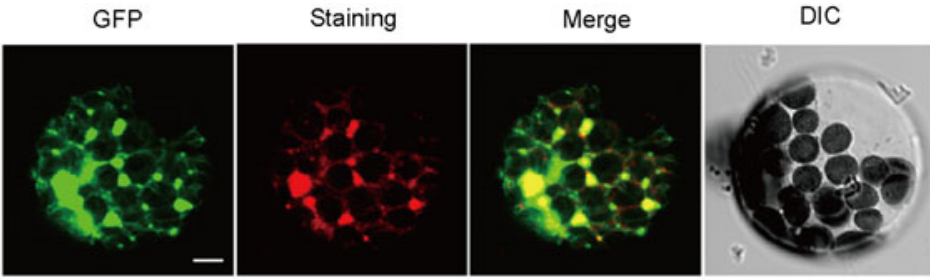

35S::GFP-AtSM34
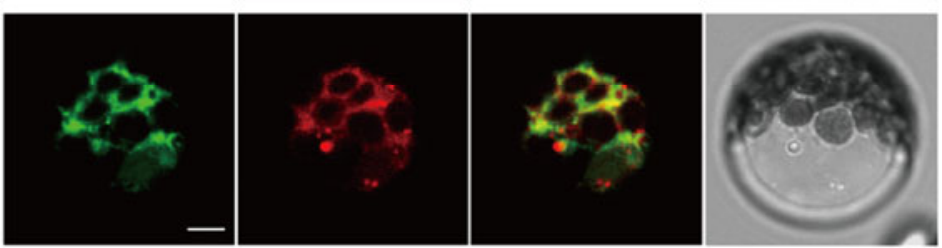

35S::AtSM34-GFP
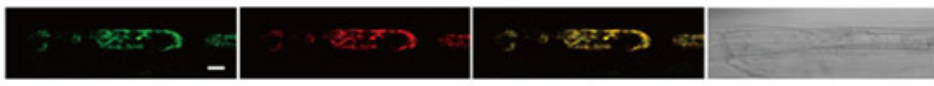

(b)

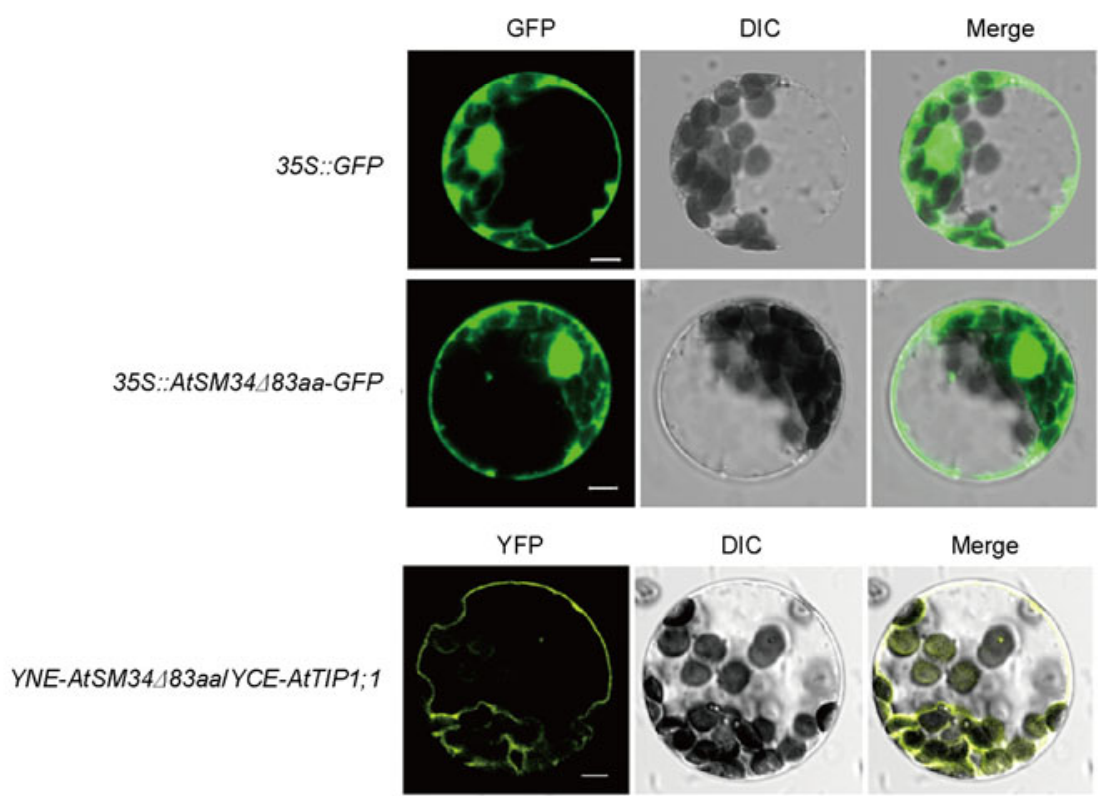

Figure 3 Subcellular localization of AtSM34 gene products in Arabidopsis mesophyll protoplasts and seeding roots. (a) Green fluorescent protein (GFP) was fused to AtSM34 at either the C-terminus (35S::AtSM34-GFP) or N-terminus (35S::GFP-AtSM34), and then transiently expressed in Arabidopsis mesophyll cell protoplasts stained with BODIPY 558/568-conjugated brefeldin A (an endoplasmic reticulum marker). Confocal imaging analysis of fluorescence signals in 35S::AtSM34-GFP transgenic Arabidopsis young root cells costained with BODIPY 558/568-conjugated brefeldin A (lower panel). (b) 35S::GFP-transformed Arabidopsis mesophyll cell protoplasts (upper panel). Protoplast transformation with AtSM34 883 aa fused to the N-terminus of GFP (middle panel). Yellow fluorescent protein (YFP) fluorescence detection of Arabidopsis mesophyll protoplasts cotransformed YNE-AtSM34 883 aa and YCE-AtTIP1;1 (bottom panel). GFP, green fluorescent protein fluorescence signals; YFP, yellow fluorescent protein fluorescence signals; DIC, differential interference contrast optics. Merge, the superimposition of both images. Scale bar $=5 \mu \mathrm{m}$. 
GFP were also observed using confocal fluorescence imaging analysis. Young root cells from stably transformed 35S::AtSM34-GFP plants and simultaneously stained with the ER marker showed overlapping green and red fluorescence, predominantly in the ER (Figure 3(a) bottom panel). This was consistent with the localization of maMYB in tobacco epidermal cells [18].

\subsection{N-terminal amino acids of AtSM34 critical for subcellular localization}

Hydrophobicity analysis predicted the presence of two transmembrane domains contained within the $83 \mathrm{~N}$-terminal amino acids of AtSM34 (http://www.ch.embnet.org/software/ TMPRED_form.html). When the GFP was fused to AtSM34 $\Delta 83 \mathrm{aa}$, the green fluorescent signals were distributed in both the cytoplasm and nucleus (Figure 3(b) middle panel). This observation indicated the N-terminal region might be essential for ER membrane subcellular localization. Ubiquitous expression of the control, 35S::GFP, was observed (Figure 3(b) upper panel).

To further elucidate the role of this region in the interaction with AtTIP1;1, Arabidopsis protoplasts were cotransformed with YNE-AtSM34 83 aa and YCE-AtTIP1;1. Restored YFP fluorescence was relocalized to the large central vacuolar membrane (Figure 3(b) bottom panel), which was similar to the tonoplast localizations of some TIP members in protoplasts [12]. This confirmed the N-terminus of AtSM34 as the transmembrane domain was not required for the protein-protein interaction.

\subsection{Hypersensitivity of AtSM34 overexpression lines to osmotic stress and ABA}

To investigate its biological function, AtSM34 was overexpressed under the control of the Super promoter and 20 independent homozygous AtSM34 overexpression lines were prepared. Quantitative real-time PCR analysis indicated the level of AtSM34 transcripts in OE-6 and OE-10 were higher than that found in the wild type (Figure 4(a)). This result was confirmed by Western blot analysis using an anti-flag antibody directed against the flag tag in the transgenic lines. Bands corresponding to the AtSM34-FLAG fusion protein were clearly visible in lines OE-6 and OE-10, whereas no expression was detected in the wild type (Figure 4(b)). Homozygotes from lines OE-6 and OE-10 were selected for use in further experiments.

To further elucidate the possible function of AtSM34, the effects of stressful conditions on seed germination and early seedling growth were investigated. Under normal growth conditions, no significant differences were observed between the transgenic and wild-type plants. However, seed germination and early growth development of transgenic plants (OE-6 and OE-10) were significantly delayed on 1/2 MS medium supplemented with high concentrations of mannitol, sorbitol or ABA (Figure 5(a)).

After $3 \mathrm{~d}$, more than $70 \%$ of the wild-type seeds germinated. In contrast, the germination rate of the transgenic seeds was less than $20 \%$ on $1 / 2$ MS medium containing 400 $\mathrm{mmol} / \mathrm{L}$ mannitol (Figure 5(b)). Similarly, on 1/2 MS medium containing $400 \mathrm{mmol} / \mathrm{L}$ sorbitol, the germination frequencies of the transgenic seeds were only $30 \%-40 \%$, whereas the germination frequencies of the wild-type seeds were $72 \%$ (Figure 5(b)). On 1/2 MS medium containing 1 $\mu \mathrm{mol} / \mathrm{L} \mathrm{ABA}$, approximately $40 \%$ of the transgenic seeds germinated after $2 \mathrm{~d}$, compared to more than $60 \%$ of the wild-type seeds. These results demonstrated that overexpression of AtSM34 conferred hypersensitivity to osmotic stress and ABA, particularly during seed germination and the early stages of plant growth.

\subsection{Expression profiles of $\mathrm{AtSM34}$ in response to stress}

To investigate the impact of osmotic stress on AtSM34 expression, 6-d-old wild-type seedlings were subjected to mannitol treatment for different periods of time and the
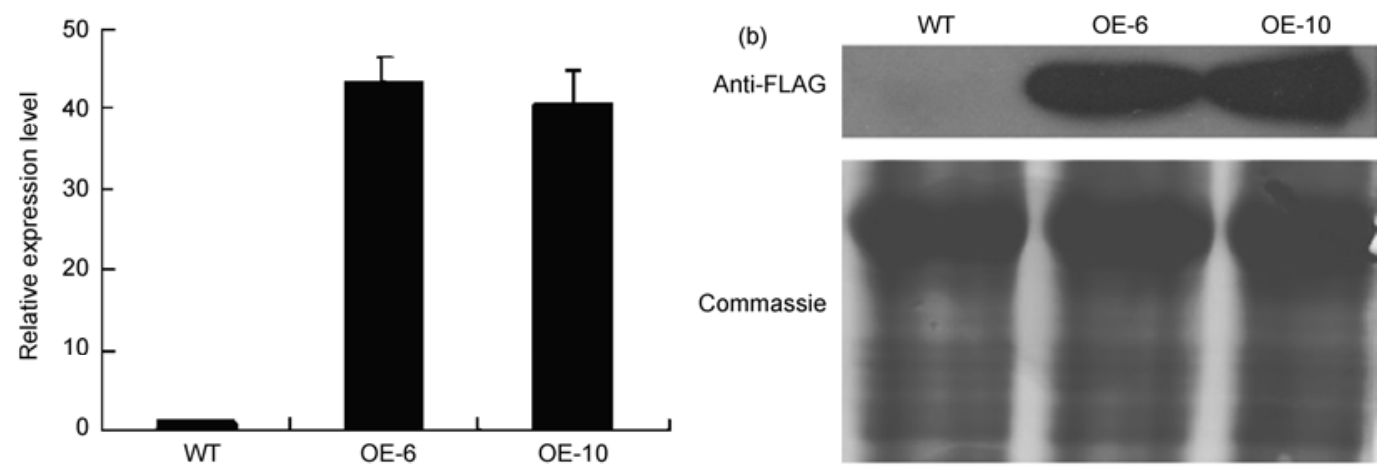

Figure 4 Analysis of AtSM34 expression levels in wild-type and overexpression line plants. (a) Quantitative real-time PCR analysis of AtSM34 transcripts in the wild-type and overexpression lines. The relative expression level of AtSM34 was analyzed by quantitative real-time PCR from 2-week-old wild-type (WT) and transgenic plants (OE-6 and OE-10) grown under normal conditions. ACTIN2/8 expression levels were analyzed as a control. (b) Western blot analysis of AtSM34 expression in WT plants and independent transgenic lines (OE-6 and OE-10). Total proteins were extracted from the mature leaves of the plants grown under normal conditions separated on $12 \%$ gel and stained with Coomassie Blue. Total proteins extracted from the WT, OE- 6 and OE-10 plants were separated on a $12 \%$ SDS-PAGE gel and followed by Western blot analysis with anti-FLAG antibodies. 
(a)
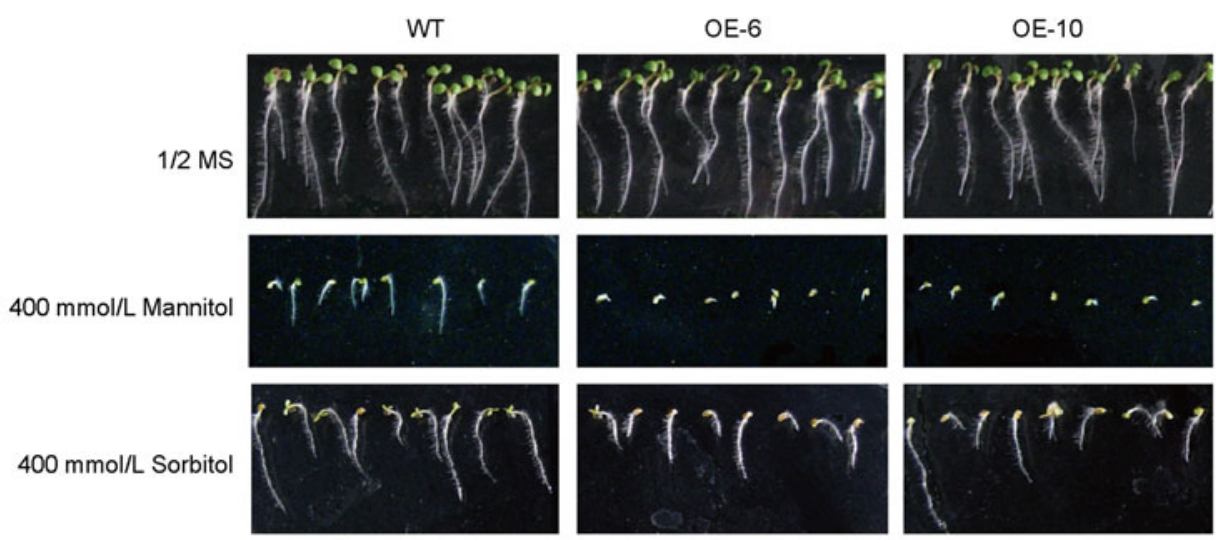

$1 \mu \mathrm{mol} / \mathrm{LABA}$
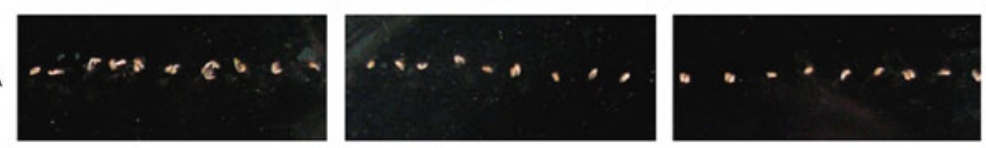

(b)
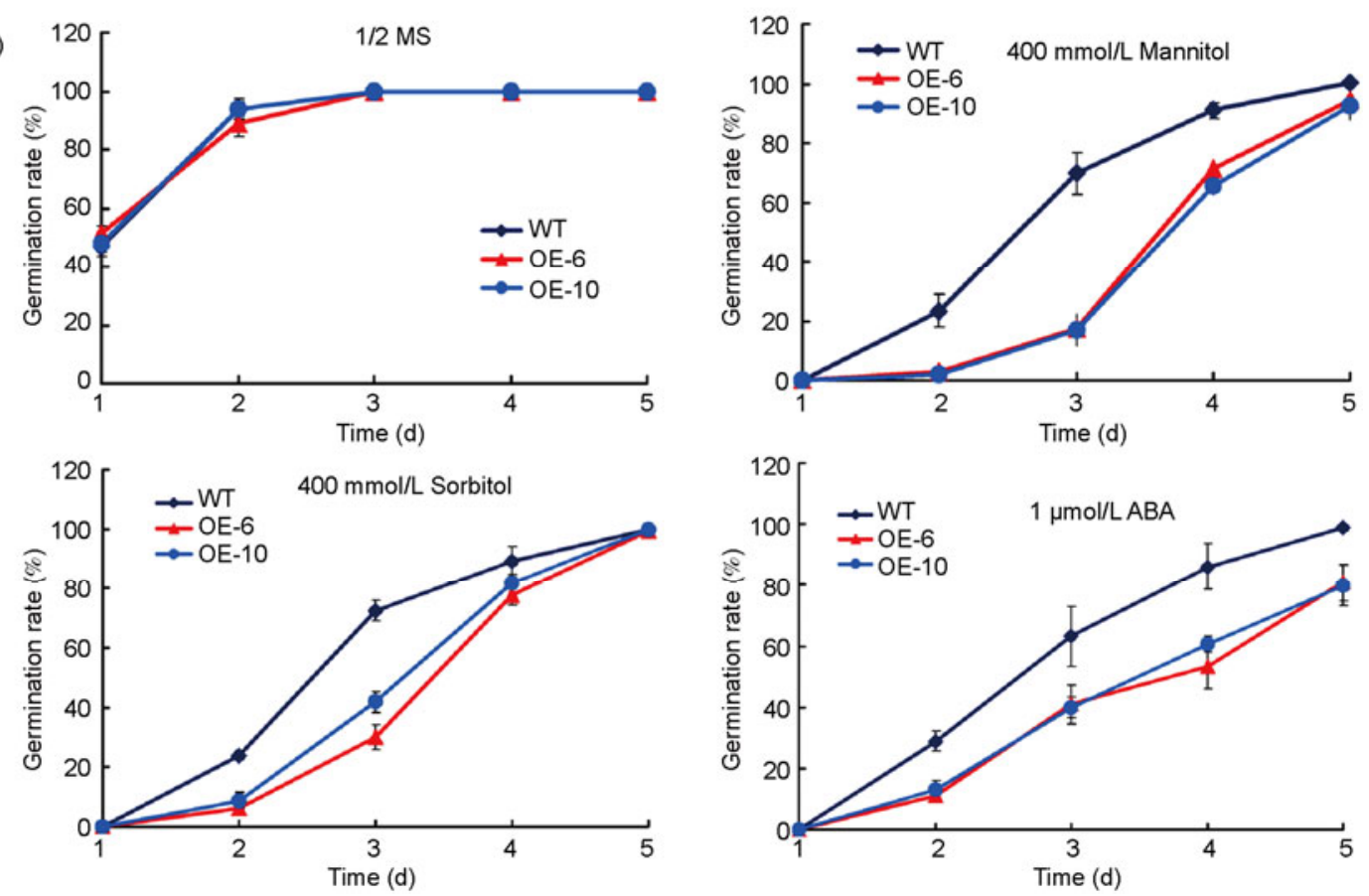

Figure 5 Overexpression of AtSM34 increases sensitivity to osmotic stress. (a) Seeds of the wild type (WT; Col-0) and AtSM34 overexpression lines (OE-6 and OE-10) were germinated on $1 / 2 \mathrm{MS}$ medium, or $1 / 2 \mathrm{MS}$ medium supplemented with $400 \mathrm{mmol} / \mathrm{L}$ mannitol, $400 \mathrm{mmol} / \mathrm{L}$ sorbitol or $1 \mu \mathrm{mol} / \mathrm{L} \mathrm{ABA}$. Photographs were taken $5 \mathrm{~d}$ after stratification or $9 \mathrm{~d}$ after stratification where seeds were grown on $1 / 2 \mathrm{MS}$ supplemented with $400 \mathrm{mmol} / \mathrm{L}$ sorbitol. (b) Effect of stress on seed germination. Germination rates of the WT and two overexpression lines (OE-6 and OE-10) seeds grown on $1 / 2$ MS medium, or on $1 / 2$ MS medium supplemented with $400 \mathrm{mmol} / \mathrm{L}$ mannitol, $400 \mathrm{mmol} / \mathrm{L}$ sorbitol or $1 \mu \mathrm{mol} / \mathrm{L}$ ABA were scored daily for $5 \mathrm{~d}$. Approximately 60 seeds from each of the WT and AtSM34 overexpression transgenic lines (OE-6 and OE-10) were used for the germination analysis in each of the media. Results are presented as the mean \pm standard deviation of three independent experiments.

expression profile of AtSM34 was analyzed by real-time PCR. Results are presented as the change in relative AtSM34 transcript abundance over time under conditions of osmotic stress (Figure 6(a)). The transcript level of AtSM34 increased and reached a maximum (about 2-fold) after 8-h treatment. However, exposure to the same conditions for 24 $\mathrm{h}$ led to a clear downregulation of AtSM34 expression.

Expression profiles of AtSM34 under other stress conditions were also analyzed by real-time PCR. Similar to the effect of osmotic stress, a slight induction in AtSM34 tran- script levels were observed following treatment with ABA, methyl viologen or glucose for 4 or $12 \mathrm{~h}$. These results indicated AtSM34 is induced in response to different types of abiotic stress and therefore might be involved in plant responses to environmental stimuli.

\subsection{Interaction of membrane-associated AtSM34 with other TIP isoforms}

The BiFC assays indicated AtSM34 interacted with the TIP 

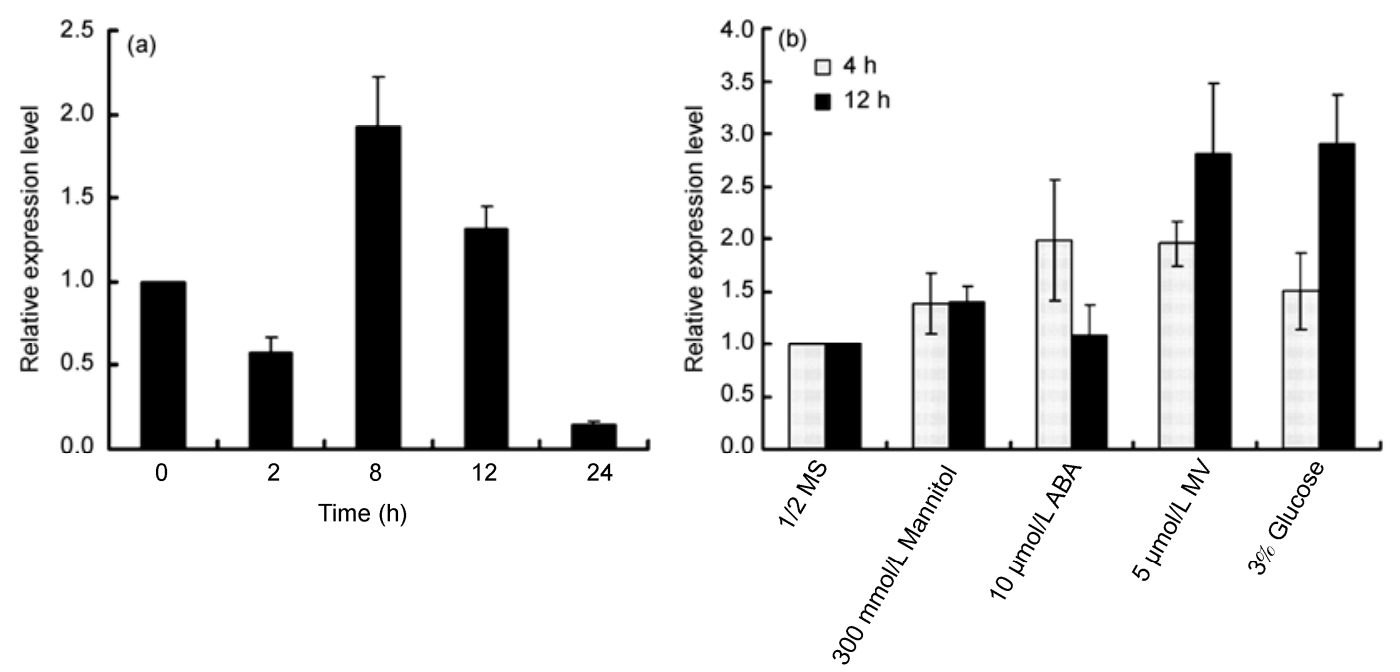

Figure 6 Expression profiles of AtSM34 in response to stress. (a) Time-course of AtSM34 expression in 6-d-old Arabidopsis seedlings grown on 1/2 MS medium supplemented with $300 \mathrm{mmol} / \mathrm{L}$ mannitol. Expression analysis was monitored by quantitative real-time RT-PCR at 0, 2, 8, 12, and 24 h. (b) Quantitative real-time RT-PCR analysis of AtSM34 transcripts grown under conditions of stress. Six-day-old seedlings were grown on $1 / 2$ MS medium supplemented with $300 \mathrm{mmol} / \mathrm{L}$ mannitol, $10 \mu \mathrm{mol} / \mathrm{L}$ ABA, $5 \mu \mathrm{mol} / \mathrm{L}$ methyl viologen (MV) or $3 \%$ glucose. Real-time PCR analyses were performed at 4 and $12 \mathrm{~h}$ using an AtSM34 gene-specific probe. ACTIN2/8 expression levels were analyzed as a control. Results are presented as the mean \pm standard deviation of three independent experiments.

isoforms, TIP1;2 and TIP2;1. Cotransformation of Arabidopsis protoplasts with YNE-AtSM34 and YCE-AtTIP1;2 or YCE-AtTIP2;1 restored YFP fluorescence with similar distribution patterns (Figure 7(a)). No YFP signal was detected with the control combinations of YCE-AtTIP1; 2/YCE-AtTIP2;1 and the YNE empty vector.

In addition, interactions between AtSM34 and the TIPs were specific. In the yeast split-ubiquitin system assays, AtSM34 interacted with AtTIP1;3, but not with AtTIP2;2 and AtTIP3;2 (Figure 7(b)). Therefore, the possibility of other aquaporins interact with AtSM34 cannot be excluded.

\subsection{Effect of AtSM34 expression of TIP genes in Ara- bidopsis under osmotic stress}

TIP1;1, TIP1;2 and TIP 2;1 are known to have the highest basal gene expression levels in tonoplast aquaporins groups 1 and 2, respectively [1], and all interacted with AtSM34 in BiFC experiments. Therefore, these isoforms were selected for further investigation of the effect of AtSM34 on aquaporin transcript levels under both normal and stress conditions. Real-time PCR analysis was performed using the TIP gene-specific primers reported in Beebo et al. [17]. As shown in Figure 8, the expression levels of TIP1;1, TIP1;2 and TIP2; 1 were modified in the overexpression lines under both normal and osmotic stress conditions. Expression levels of the three TIPs were downregulated in the wild type and OE-6 and OE-10, with rapid downregulation observed in the $\mathrm{OE}$ lines under osmotic stress for $12 \mathrm{~h}$. These results demonstrated ectopic expression of AtSM34 disrupts the natural expression patterns of some TIP genes in Arabidopsis plants under osmotic stress.

\section{Discussion}

In this study, we identified a novel protein, AtSM34, as a putative interactor with members of the Arabidopsis tonoplast intrinsic protein family by experiments in yeast and plant cells. Although there are a number of reports of aquaporin-interacting proteins in mammalian systems, there are very few reports in plants, with the exception of some aquaporins known to form homomers or heteromers [35]. TIPs are one of the most abundant aquaporin subgroups in plants and, as such, the detection of their interrelated proteins may elucidate the mechanisms of their function and regulation.

It is known that all aquaporin isoforms traffic through the secretory pathway before reaching their final destination compartments during the course of their synthesis and maturation. Reports have indicated expression of TIPs is predominantly located in the tonoplast [12]. However, McTIP1;2 (McMIPF) proteins redistributed to the endosomes in osmotic stress [14]. Recent studies have indicated that aquaporin trafficking is a critical point for regulating aquaporin expression and function [35]. The results of BiFC colocalization suggested that the interactions might occur in small vesicles that are involved in the process of the aquaporin sorting between ER and vacuoles although the mechanism of this process requires further investigation. Changes in the interaction patterns after deletion of the first 83 aa could be due to the deletion of the TM domains of AtSM34, making it cytosolic, thus, able to directly interact with any cytosolic facing domain of TIP1;1. AtSM34 $\Delta 83$ is soluble and thus synthesized by cytosolic ribosomes and does not depend on the secretory pathway anymore. Therefore, their interaction occurred in the vacuole membrane, which was 

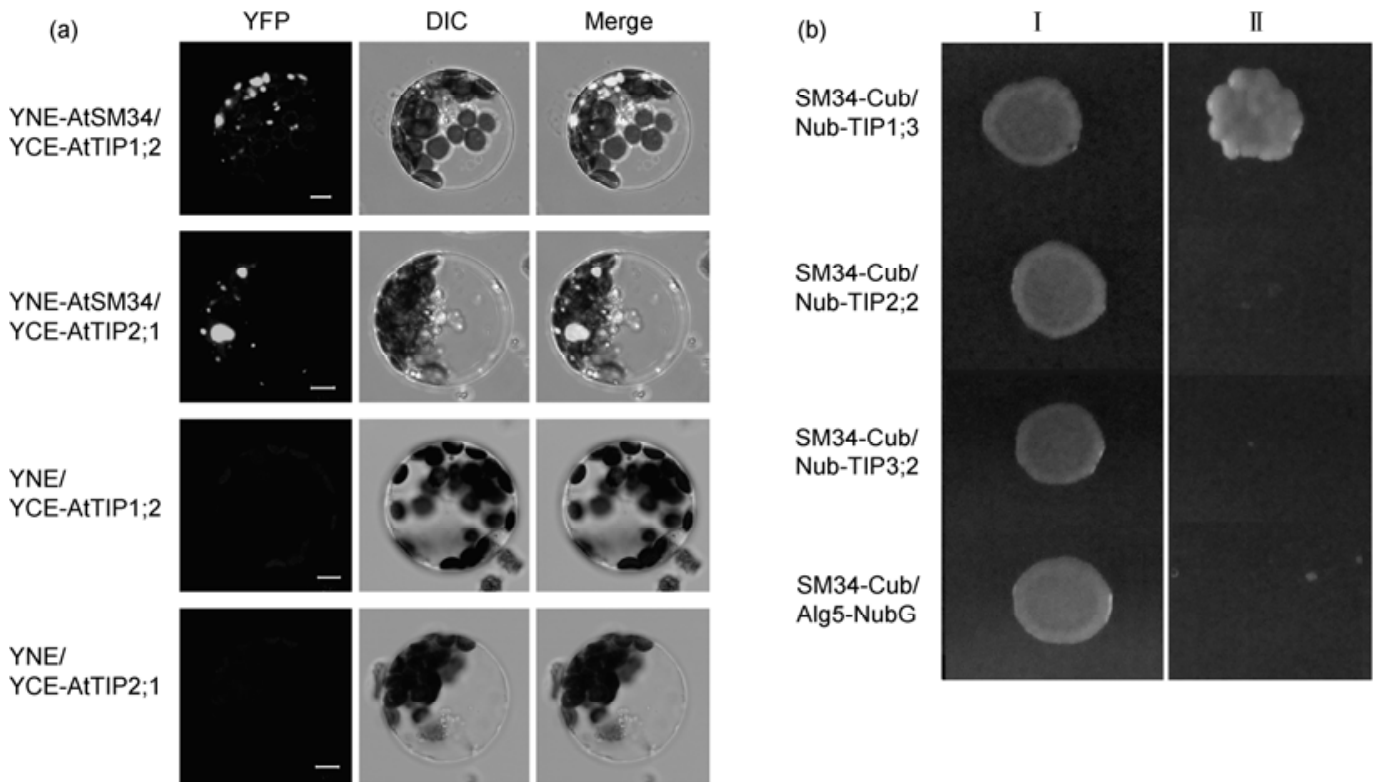

III

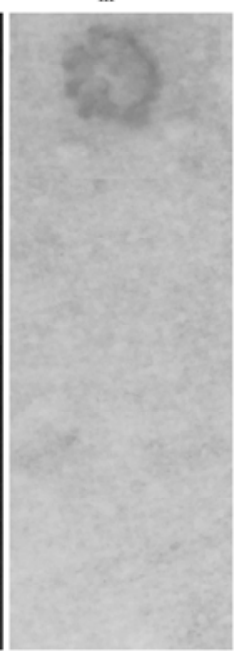

Figure 7 Analysis of interactions between AtSM34 and other TIP isoforms. (a) Bimolecular fluorescence complementation assay demonstrating the interactions between AtSM34 and the TIP isoforms, AtTIP1;2 or AtTIP2;1. YFP, yellow fluorescent protein fluorescence signals; DIC, differential interference contrast optics; Merge, the superimposition of both images. Scale bar $=5 \mu \mathrm{m}$. (b) Yeast split-ubiquitin system assay showing AtSM34 interaction with other TIPs in yeast. The combination of AtSM34-Cub/pAlg5-NubG was used as a negative control. I, synthetic dropout selection medium that lacked both Trp and Leu (SD/-Trp-Leu); II, synthetic dropout selection medium that lacked Trp, Leu and His (SD/-Trp-Leu-His) supplemented with $10 \mathrm{mmol} / \mathrm{L}$ 3-AT; III, $\beta$-galactosidase activity assay.
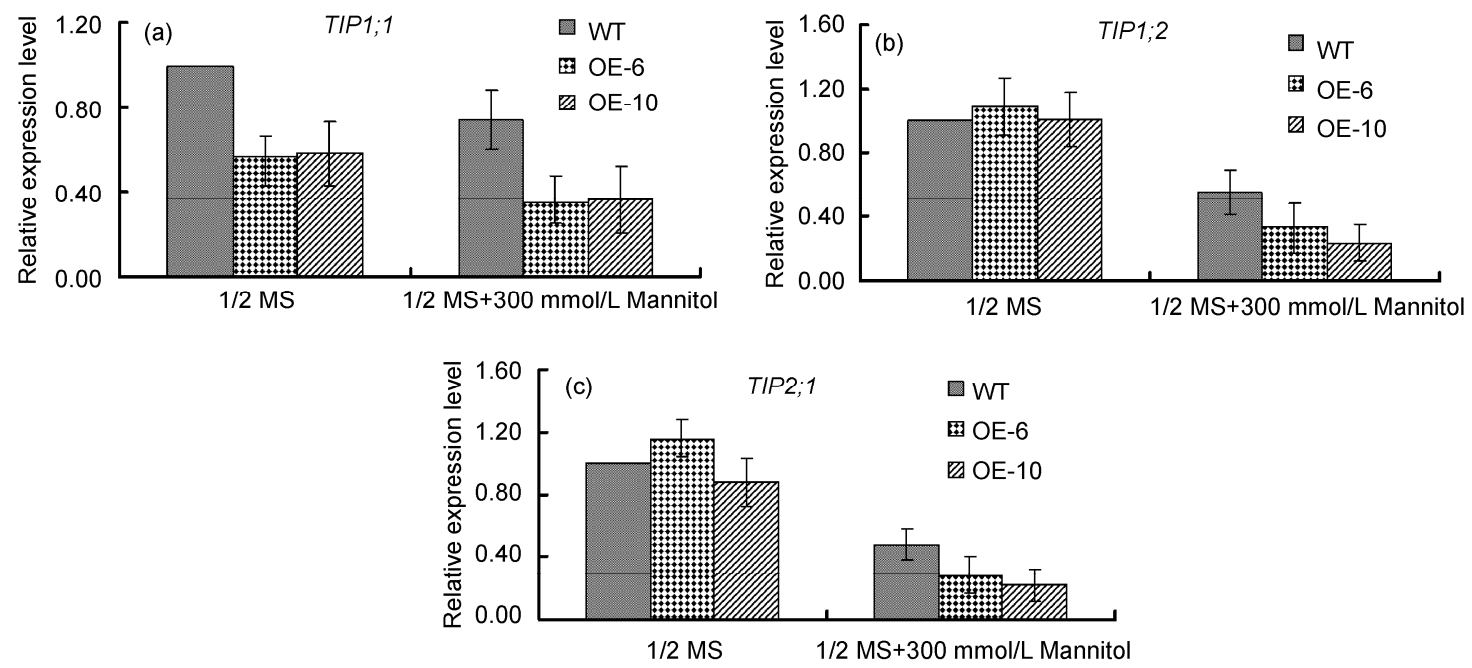

Figure 8 Expression profiles of AtTIP genes in Arabidopsis plants under osmotic stress. The expression of TIP1;1, TIP1;2, and TIP2;1 in the wild-type (WT; Col-0) and AtSM34 transgenic overexpressing lines (OE-6 and OE-10) in response to osmotic stress was measured by quantitative real-time PCR. Arabidopsis plants were grown in 1/2 MS medium for $6 \mathrm{~d}$ followed by further incubation for $12 \mathrm{~h}$ in $1 / 2 \mathrm{MS}$ liquid medium supplemented with $300 \mathrm{mmol} / \mathrm{L}$ mannitol. Plots represent the relative expression (fold changes) of each gene in transgenic plants (OE-6 and OE-10) compared with expression in the WT plants. ACTIN2/8 expression levels were analyzed as a control. Results are presented as the mean \pm standard deviation of three independent experiments.

identical to the observed TIP1;1 localization.

In AtSM34 promoter controlled $\beta$-glucuronidase (GUS) transgenic plants, and strong GUS signals were observed in cotyledons of the germinating seeds (Figure 2(a)) and seedlings (Figure 2(b), (c)). This indicated that AtSM34 might be involved in seed germination and early seedling growth.

A role for aquaporins in cell osmoregulation and maturation of the vacuolar apparatus during late seed development and during the early stages of germination has been proposed [15]. This hypothesis is based on the observation that tissue water content alters significantly during seed maturation and germination and knowledge of the expression and regulation properties of tonoplast aquaporins ( $\alpha$-TIP and $\beta$-TIP, or TIP3s) that have been detected in seeds $[36,37]$. Initial expression of aquaporins has been correlated with germination efficiency [38]. During growth and develop- 
ment, tonoplast aquaporins modulate the tonoplast permeability and mediate transcellular water transport. In this way, aquaporins contribute to water imbibition in seed germination and facilitate water supply to the expanding tissues for cell enlargement [38,39]. The expression profiles of AtTIPs genes obtained by quantitative real-time PCR revealed that the levels of $T I P 1 ; 1, T I P 1 ; 2$ and TIP2;1 transcripts are lower in OE lines under conditions of osmotic stress. Therefore, we proposed that the transgenic plants absorb water more slowly than the wide-type, resulting in a delayed rate of seed germination. In addition, at least 10 homologous TIP isoforms have been identified in Arabidopsis. The possibility that other AQPs interact with AtSM34, leading to delayed germination in response to osmotic stress in the early stage of growth cannot be excluded.

To gain further insight into the role of AtSM34 in the response to osmotic stress, the expression of marker genes known to be involved in regulating this response were monitored by real-time PCR analysis. These marker genes included RD22, RD29A, DREB2A, COR47, KIN1, KIN2, ERD10, NCED3, ADH1 and bZIP60. No significant alteration in the expression of these genes was detected, with the exception of a decrease in RD29A in the AtSM34 OE lines under conditions of osmotic stress for $12 \mathrm{~h}$ (Figure S1). $R D 29 A$ has been reported to have at least two cis-acting elements; one involved in the ABA-associated response to desiccation and the other induced by changes in osmotic potential [40]. These data indicated that AtSM34 is not involved in the usual stress signaling pathway.

In plants, the MYB family has selectively expanded and MYB proteins are key factors in regulatory networks controlling development, metabolism and responses to biotic and abiotic stresses [19,41-44]. Novel proteins harboring Myb/SANT-like domains distinct from other described MYB-like transcriptional regulators have been detected in plants gradually. Ectopic expression of the tomato early fruit specific gene, Lefsm1, resulted in severe developmental alterations, retarded growth, and reduced apical dominance during tomato and Arabidopsis seedling development [45]. $M Y B$ related genes have been implicated in a wide variety of other plant-specific processes, although their physiological function has not been fully understood, and characterization of a great number of MYB-like proteins remains elusive [46]. So, AtSM34 is likely to be a potential membrane-tethered transcription factor and the possibility remains that AtSM34 interacts with other proteins in addition to aquaporins to delay germination of AtSM34 overexpressing plants under conditions of osmotic stress cannot be excluded.

The precise function of AtSM34 in the response of plants to osmotic stress at the early growth stage remains to be characterized. Further studies of downstream targets of AtSM34 will provide a greater understanding of the molecular function of this protein.
This work was supported by the National Natural Science Foundation of China (30770193), the National Basic Research Program of China (2006CB100100), and the "111 Project" (B06003).

1 Alexandersson E, Fraysse L, Sjovall-Larsen S, et al. Whole gene family expression and drought stress regulation of aquaporins. Plant Mol Biol, 2005, 59: 469-484

2 Jang J Y, Rhee J Y, Kim D G, et al. Ectopic expression of a foreign aquaporin disrupts the natural expression patterns of endogenous aquaporin genes and alters plant responses to different stress conditions. Plant Cell Physiol, 2007, 48: 1331-1339

3 Gerbeau P, Amodeo G, Henzler T, et al. The water permeability of Arabidopsis plasma membrane is regulated by divalent cations and pH. Plant J, 2002, 30: 71-81

4 Uehlein N, Lovisolo C, Siefritz F, et al. The tobacco aquaporin NtAQP1 is a membrane $\mathrm{CO}_{2}$ pore with physiological functions. Nature, 2003, 425: 734-737

5 Chaumont F, Moshelion M, Daniels M J. Regulation of plant aquaporin activity. Biol Cell, 2005, 97: 749-764

6 Fischer M, Kaldenhoff R. On the $\mathrm{pH}$ regulation of plant aquaporins. J Biol Chem, 2008, 283: 33889-33892

7 Reichow S L, Gonen T. Noncanonical binding of calmodulin to aquaporin-0: Implications for channel regulation. Structure, 2008, 16: 1389-1398

8 Lu H A, Sun T X, Matsuzaki T, et al. Heat shock protein 70 interacts with aquaporin-2 and regulates its trafficking. J Biol Chem, 2007, 282: 28721-28732

9 Kim M J, Kim H R, Paek K H. Arabidopsis tonoplast proteins TIP1 and TIP2 interact with the cucumber mosaic virus 1a replication protein. J Gen Virol, 2006, 87: 3425-3431

10 Masalkar P, Wallace I S, Hwang J H, et al. Interaction of cytosolic glutamine synthetase of soybean root nodules with the C-terminal domain of the symbiosome membrane nodulin 26 aquaglyceroporin. J Biol Chem, 2010, 285: 23880-23888

11 Lee H K, Cho S K, Son O, et al. Drought stress-induced Rma1H1, a RING membrane-anchor E3 ubiquitin ligase homolog, regulates aquaporin levels via ubiquitination in transgenic Arabidopsis plants. Plant Cell, 2009, 21: 622-641

12 Wudick M M, Luu D T, Maurel C. A look inside: Localization patterns and functions of intracellular plant aquaporins. New Phytol, 2009, 184: 289-302

13 Kaldenhoff R, Fischer M. Aquaporins in plants. Acta Physiol (Oxf), 2006, 187: 169-176

14 Vera-Estrella R, Barkla B J, Bohnert H J, et al. Novel regulation of aquaporins during osmotic stress. Plant Physiol, 2004, 135: 2318-2329

15 Maurel C, Chrispeels M, Lurin C, et al. Function and regulation of seed aquaporins. J Exp Bot, 1997, 48: 421-430

16 Schüssler M D, Alexandersson E, Bienert G P, et al. The effects of the loss of TIP1;1 and TIP1;2 aquaporins in Arabidopsis thaliana. Plant J, 2008, 56: 756-767

17 Beebo A, Thomas D, Der C, et al. Life with and without AtTIP1;1, an Arabidopsis aquaporin preferentially localized in the apposing tonoplasts of adjacent vacuoles. Plant Mol Biol, 2009, 70: 193-209

18 Slabaugh E, Held M, Brandizzi F. Control of root hair development in Arabidopsis thaliana by an endoplasmic reticulum anchored member of the R2R3-MYB transcription factor family. Plant J, 2011, DOI: 10.1111/j.1365-313X.2011.04602.X

19 Stracke R, Werber M, Weisshaar B. The R2R3-MYB gene family in Arabidopsis thaliana. Curr Opin Plant Biol, 2001, 4: 447-456

20 Walter M, Chaban C, Schutze K, et al. Visualization of protein interactions in living plant cells using bimolecular fluorescence complementation. Plant J, 2004, 40: 428-438

21 Sheen J. A transient expression assay using Arabidopsis mesophyll protoplasts. 2002, http://genetics.mgh.harvard.edu/sheenweb/

22 An R, Chen Q J, Chai M F, et al. AtNHX8, a member of the monovalent cation: Proton antiporter-1 family in Arabidopsis thaliana, encodes a putative Li/H antiporter. Plant J, 2007, 49: 718-728

$23 \mathrm{Xu} \mathrm{J}, \mathrm{Li} \mathrm{H} \mathrm{D,} \mathrm{Chen} \mathrm{L} \mathrm{Q,} \mathrm{et} \mathrm{al.} \mathrm{A} \mathrm{protein} \mathrm{kinase,} \mathrm{interacting} \mathrm{with} \mathrm{two}$ 
calcineurin B-like proteins, regulates $\mathrm{K}^{+}$transporter AKT1 in Arabidopsis. Cell, 2006, 125: 1347-1360

24 Cao X, Yang K Z, Xia C, et al. Characterization of DUF724 gene family in Arabidopsis thaliana. Plant Mol Biol, 2010, 72: 61-73

25 Wang M, Xu Q, Yu J, et al. The putative Arabidopsis zinc transporter ZTP29 is involved in the response to salt stress. Plant Mol Biol, 2010, 73: 467-479

26 Chen N Z, Zhang X Q, Wei P C, et al. AtHAP3b plays a crucial role in the regulation of flowering time in Arabidopsis during osmotic stress. J Biochem Mol Biol, 2007, 40: 1083-1089

27 Charrier B, Champion A, Henry Y, et al. Expression profiling of the whole Arabidopsis shaggy-like kinase multigene family by real-time reverse transcriptase-polymerase chain reaction. Plant Physiol, 2002, 130: $577-590$

28 Livak K J, Schmittgen T D. Analysis of relative gene expression data using real-time quantitative PCR and the $2^{\text {(-Delta Delta } \mathrm{C}(\mathrm{T}))}$ method. Methods, 2001, 25: 402-408

29 Chen Y F, Li L Q, Xu Q, et al. The WRKY6 transcription factor modulates PHOSPHATE1 expression in response to low Pi stress in Arabidopsis. Plant Cell, 2009, 21: 3554-3566

30 Clough S J, Bent A F. Floral dip: A simplified method for Agrobacterium-mediated transformation of Arabidopsis thaliana. Plant $\mathrm{J}$, 1998, 16: 735-743

31 Hunter P R, Craddock C P, Di Benedetto S, et al. Fluorescent reporter proteins for the tonoplast and the vacuolar lumen identify a single vacuolar compartment in Arabidopsis cells. Plant Physiol, 2007, 145: 1371-1382

32 Vitale A, Hinz G. Sorting of proteins to storage vacuoles: How many mechanisms? Trends Plant Sci, 2005, 10: 316-323

33 Jelinkova A, Malinska K, Simon S, et al. Probing plant membranes with FM dyes: Tracking, dragging or blocking? Plant J, 2010, 61: 883-892

34 Kim D H, Eu Y J, Yoo C M, et al. Trafficking of phosphatidylinositol 3-phosphate from the trans-Golgi network to the lumen of the central vacuole in plant cells. Plant Cell, 2001, 13: 287-301

35 Maurel C, Verdoucq L, Luu D T, et al. Plant aquaporins: Membrane channels with multiple integrated functions. Annu Rev Plant Biol,
2008, 59: 595-624

36 Johnson K D, Herman E M, Chrispeels M J. An abundant, highly conserved tonoplast protein in seeds. Plant Physiol, 1989, 91: $1006-1013$

37 Höfte H, Hubbard L, Reizer J, et al. Vegetative and seed-specific forms of tonoplast intrinsic protein in the vacuolar membrane of Arabidopsis thaliana. Plant Physiol, 1992, 99: 561-570

38 Vander W C, Postaire O, Tournaire-Roux C, et al. Expression and inhibition of aquaporins in germinating Arabidopsis seeds. Plant Cell Physiol, 2006, 47: 1241-1250

39 Peng Y, Lin W, Cai W, et al. Overexpression of a Panax ginseng tonoplast aquaporin alters salt tolerance, drought tolerance and cold acclimation ability in transgenic Arabidopsis plants. Planta, 2007, 226: $729-740$

40 Yamaguchi-Shinozaki K, Shinozaki K. Characterization of the expression of a desiccation-responsive $r d 29$ gene of Arabidopsis thali$a n a$ and analysis of its promoter in transgenic plants. Mol Gen Genet, 1993, 236: 331-340

41 Lee M M, Schiefelbein J. Developmentally distinct MYB genes encode functionally equivalent proteins in Arabidopsis. Development, 2001, 128: 1539-1546

42 Abe H, Urao T, Ito T, et al. Arabidopsis AtMYC2 (bHLH) and AtMYB2 (MYB) function as transcriptional activators in abscisic acid signaling. Plant Cell, 2003, 15: 63-78

43 Denekamp M, Smeekens S C. Integration of wounding and osmotic stress signals determines the expression of the AtMYB102 transcription factor gene. Plant Physiol, 2003, 132: 1415-1423

44 Araki S, Ito M, Soyano T, et al. Mitotic cyclins stimulate the activity of c-Myb-like factors for transactivation of G2/M phase-specific genes in tobacco. J Biol Chem, 2004, 279: 32979-32988

45 Barg R, Sobolev I, Eilon T, et al. The tomato early fruit specific gene Lefsm1 defines a novel class of plant-specific SANT/MYB domain proteins. Planta, 2005, 221: 197-211

46 Hamaguchi A, Yamashino T, Koizumi N, et al. A small subfamily of Arabidopsis RADIALIS-LIKE SANT/MYB genes: A link to HOOKLESS1-mediated signal transduction during early morphogenesis. Biosci Biotechnol Biochem, 2008, 72: 2687-2696

Open Access This article is distributed under the terms of the Creative Commons Attribution License which permits any use, distribution, and reproduction in any medium, provided the original author(s) and source are credited.

\section{Supporting Information}

Figure S1 Expression level of stress marker gene RD29A in WT and overexpressing lines (OE-6 and OE-10) under conditions of osmotic stress.

The supporting information is available online at csb.scichina.com and www.springerlink.com. The supporting materials are published as submitted, without typesetting or editing. The responsibility for scientific accuracy and content remains entirely with the authors. 